\title{
Hemosiderosis pulmonar idiopática: seguimiento de pacientes durante 25 años (1995-2019)
}

\author{
JULIO MAGGIOLO M.*, LILIAN RUBILAR O.* y GUIDO GIRARDI B.**
}

\section{Idiopathic pulmonary hemosiderosis: follow-up of patients for 25 years (1995-2019)}

Idiopathic pulmonary hemosiderosis (IPH) is a cause of diffuse alveolar hemorrhage. Objective: to describe the evolution of children with IPH in our institution. Retrospective monitoring with a follow-up protocol was carried out. 13 patients, seven males, were recruited. From an agricultural area (6/13). Not all of patients had the complete diagnostic triad: cotton infiltrates (9/13), anemia (11/13), hemoptysis (9/13). Hemosiderin-laden macrophages counting in the bronchoalveolar lavage fluid was over $30 \%$ in all the patients. Computed chest tomography was informed as normal (5/13), interstitial pattern (5/13), ground glass (2/13) and fibrosis (1/13). Spirometry: normal (7/13), restrictive (4/13), obstructive (1/13) and not performed (1/13). Treatment during the acute phase: bolus of methylprednisolone (7/13) or prednisone (6/13) or hydrocortisone (1/13). In the maintenance phase: prednisone (13/13) plus an immunosuppressant, azathioprine (12/13), hydroxychloroquine (1/13), mycophenolate (1/13), plus budesonide MDI (13/13). Eight patients stopped the bleeding episodes. Two patients died and there were five physiological pregnancies in 3 adolescents. It was observed:(a) different modes of IPH presentation that delayed its diagnosis; (b) large exposure to pesticides; (c) prognosis improved if diagnosis and treatment were early, also in adolescent girls; (d) most of the patients stopped the bleeding episodes.

Key words: Hemosiderosis; hemoptisis; anemia; hemosiderin; macrophages; corticosteroids; immunosuppressive agents.

\section{Resumen}

La hemosiderosis pulmonar idiopática (HPI) es una causa de hemorragia alveolar difusa. Objetivo: describir la evolución de niños con HPI en nuestra institución. Se realizó una revisión retrospectiva con protocolo de seguimiento. Se reclutaron 13 pacientes, 7 hombres. Procedentes de una zona agrícola (6/13). No todos presentaron la tríada diagnóstica completa: infiltrados algodonosos (9/13), anemia (11/13), hemoptisis (9/13). Todos evidenciaron un recuento de hemosiderófagos sobre $30 \%$ en el lavado broncoalveolar. Tomografía computada de tórax: normal (5/13), patrón intersticial (5/13), vidrio esmerilado (2/13) y fibrosis (1/13). Espirometría: normal (7/13), restrictiva (4/13), obstructiva (1/13) y no efectuada (1/13). Tratamiento durante la fase aguda: bolos de metilprednisolona (7/13) o prednisona (6/13) o hidrocortisona (1/13). En la fase de mantención se administró: prednisona (13/13) más un inmunosupresor, azathioprina (12/13), hidroxicloroquina (1/13), micofenolato (1/13), más budesonida MDI (13/13). Ocho pacientes detuvieron los sangrados. Dos pacientes fallecieron y hubo cinco embarazos de curso fisiológico en 3 adolescentes. Se observó: a) diferentes modalidades de presentación que retrasaron el diagnóstico; b) gran exposición a pesticidas; c) mejor pronóstico si el diagnóstico y el tratamiento eran precoces, también en niñas adolescentes; d) la mayoría detuvo los episodios de sangrado.

Palabras clave: Hemosiderosis; hemoptisis; anemia; hemosiderófagos; corticoides; agente inmunosupresores.

* Pediatra, Especialista en Enfermedades Respiratorias. Unidad Broncopulmonar. Hospital Exequiel González Cortés. Santiago, Chile.

** Pediatra, Especialista en Enfermedades Respiratorias. Profesor Titular. Universidad de Chile. Campus Sur. Santiago, Chile. 


\section{Introducción}

La hemosiderosis pulmonar idiopática (HPI) es una patología poco frecuente, se caracteriza por presentar hemorragia alveolar difusa (HAD), potencialmente fatal ${ }^{1-8}$. La mayoría de los casos se presenta en la edad pediátrica, durante la primera década de la vida, sin predilección de género ${ }^{1-3}$. Esta condición ocurre en ausencia de enfermedades sistémicas y de otras patologías asociadas a hemorragia pulmonar ${ }^{1-8}$.

La clásica tríada diagnóstica consiste en anemia, hemoptisis e infiltrados pulmonares, no siempre se presenta completa ${ }^{1-7}$. La hemoptisis puede ser masiva conduciendo a shock hipovolémico y anemia intensa, con eventual riesgo vital, en cambio, la pérdida crónica de sangre se puede presentar sin hemoptisis, si la hemorragia no es controlada, conducirá a anemia ferropriva resistente a la terapia con hierro, lo que puede producir latencia en el diagnóstico, además el paciente puede evolucionar a una enfermedad pulmonar intersticial, insuficiencia respiratoria crónica y finalmente fibrosis pulmonar.

La radiografía $(\mathrm{Rx})$ de tórax puede mostrar en la fase aguda imágenes algodonosas, confundiéndose con neumonía, lo que también puede retrasar el diagnóstico ${ }^{8-10}$. La tomografía computada (TC) de tórax inicialmente puede evidenciar infiltrados algodonosos, además de vidrio esmerilado y posteriormente infiltrados intersticiales y micronódulos difusos ${ }^{9,10}$. El diagnóstico requiere la presencia de $30 \%$ de hemosiderófagos (HSF), la muestra debe ser obtenida de elección mediante lavado broncoalveolar (LBA), pero también de contenido gástrico o esputo inducido ${ }^{11-13}$. Esta enfermedad muestra recurrencias, lo que obliga a mantener un tratamiento continuo a largo plazo, con corticoides e inmunosupresores, esquema que ha mejorado el pronóstico y la calidad de vida, incluso se ha reportado recuperación completa $^{14-20}$. La posibilidad de fallecer, aún con tratamiento, está presente. Se ha usado inmunoglobulina en dosis inmunomoduladoras ${ }^{2-4}$, sin evidentes beneficios.

El trasplante pulmonar no se recomienda, debido a que se ha reportado recurrencias en el órgano donante ${ }^{21}$.

\section{Objetivo general}

Presentar el diagnóstico y la evolución de pacientes con HPI entre los años 1995 a 2019.

\section{Objetivos específicos}

Describir las diferentes modalidades de presentación, la evolución con los esquemas terapéuticos usados y las reacciones adversos a medicamentos (RAM) en el período de tiempo mencionado.

\section{Pacientes y Métodos}

Estudio retrospectivo con protocolo de seguimiento de pacientes con HPI, diagnosticados en la Sección de Neumología Pediátrica, Hospital de Niños Dr. Exequiel González Cortés, Santiago de Chile, reclutados desde 1995 a 2019.

1. Para llegar al diagnóstico de HPI, se consideró la clínica, hemograma, Radiografía (Rx) y tomografía computada (TC) de tórax y recuento de HSF en LBA. Con estos elementos se diagnosticó una hemorragia alveolar difusa (HAD). Posteriormente, durante el seguimiento, se efectuó el descarte de causas conocidas de HAD y de esta forma se concluyó el diagnóstico de HPI.

2. Para el seguimiento se realizó lo siguiente:

a) Estudio básico: hemograma; Rx tórax, TC tórax; HSF en LBA; espirometrías (MED-GRAPHIC); pletismografía (MEDGRAPHIC).

b) Exámenes para descartar otras causas de HAD: electrocardiograma, ecocardiograma, examen de orina, creatininemia, complemento, factor reumatoide, anticuerpos antimembrana basal glomerular, anticuerpos anticitoplasma de neutrófilos (ANCA), anticuerpo antinuclear, anticuerpos antifosfolípidos, inmunoglobulina (Ig) $\mathrm{G}$ e IgE antiproteína de leche de vaca, anticuerpo antigliadina y antitransglutaminasa.

c) Pruebas para descartar otras causas de anemia y trastornos de la coagulación.

d) Exámenes para evaluar las RAM: bilirrubina, transaminasas, glicemia, calcemia y fosfemia. Evaluación oftalmológica. Rx huesos largos o densitometría.

3. Tratamiento

Fase aguda: metilprednisolona bolos (10-15 $\mathrm{mg} / \mathrm{kg} /$ dosis por 3 días, máximo $1 \mathrm{~g} /$ dosis) o prednisona $(2 \mathrm{mg} / \mathrm{kg} /$ día por 5 días $)$ o hidrocortisona (10 mg/kg/día por 5 días).

Fase de mantención: prednisona (1-2 mg/kg/ día), más azatioprina (3-5 mg/kg/día) o hidroxicloroquina $(10 \mathrm{mg} / \mathrm{kg} /$ día $)$ o micofenolato (600 mg/m²/12 h; dosis máxima: $2 \mathrm{~g} /$ día), más budesonida MDI (800 - $1.600 \mu \mathrm{g} /$ día).

El presente artículo cuenta con la aprobación del Comité de Ética de nuestro hospital. 


\section{Resultados}

De enero de 1995 a diciembre de 2019 fueron reclutados 13 pacientes, 7 hombres. El diagnóstico inicial fue neumonía (8/13), edema pulmonar agudo (2/13), anemia (4/13), lo que produjo latencia en el diagnóstico de HPI, que en promedio fue de 2,3 años. Presentaron hemoptisis (9/13).

En todos se obtuvo recuentos de HSF sobre $30 \%$ en el LBA, luego del cuarto día del sangrado. Seis pacientes provenían de una misma zona rural con intenso uso de pesticidas. En todos los pacientes se descartaron otras causas de HAD.

TC tórax: patrón normal $(5 / 13)$, intersticial $(5 / 13)$, vidrio esmerilado (2/13), fibrosis (1/13). Espirometría: normal (7/13), patrón restrictivo $(4 / 13)$, obstructivo (1/13), en 1 no se logró realizar.

Durante la fase aguda al comienzo del seguimiento se prescribió a un paciente hidrocortisona, siendo refractario a la terapia, por lo que se cambió a metilprednisolona una dosis diaria durante 3 días consecutivos, la que se usó en otros 6 pacientes, todos dejaron de sangrar al segundo día de tratamiento. Cuando fue usada prednisona (6/13), también hubo buena respuesta, sin embargo, con mayor latencia.

Durante la fase de mantención se continuó con prednisona permanente más un inmunosupresor. Se usó azatioprina (12/13), hidroxicloroquina $(1 / 13)$, dos pacientes mantuvieron el sangrado, por lo que se cambió la azathioprina por hidroxicloroquina, y en otro, por falta de respuesta a hidroxicloroquina y a azathioprina, se cambió por micofenolato, con buenos resultados. En todos los pacientes se agregó budesonida MDI (13/13).

Las RAM observadas fueron: síndrome de Cushing (10/13), osteoporosis (5/13), opacidades subcapsulares $(3 / 13)$, diabetes por corticoides $(1 / 13)$.

Seis pacientes presentaron recaídas serias, 2 necesitaron ventilación mecánica invasiva (VMI), 1 ventilación mecánica no invasiva (VMNI) y 1 ventilación mecánica de alta frecuencia (VAFO). Algunas recaídas estuvieron asociadas a interrupción del tratamiento.

2/13 fallecieron, debido a hemorragias intratables, un paciente por suspensión del tratamiento y otra enferma por una neumonía bacteriana grave.

Los 8 pacientes mejorados llevan 1 a 17 años de evolución, con un promedio de 8 años, sin recaídas. El rango de edad es de 17 a 33 años.

Tres pacientes presentaron cinco embarazos sin incidentes, los partos fueron resueltos vía cesárea, con hijos sanos, todas presentaron escasos sangrados durante el puerperio. Una niña se embarazó estando en tratamiento, otra presentó 3 embarazos sucesivos luego de haber suspendido 2 años la terapia y la tercera paciente luego de 4 años sin tratamiento. La Tabla 1 muestra los datos demográficos, clínicos, radiológicos, evolución, función pulmonar y tratamiento.

\section{Discusión}

La HPI es una de las causas de HAD, sin capilaritis y de causa desconocida, es poco frecuente, presenta una incidencia de 0,24 (Suecia) y 1,23 (Japón) casos/millón niños/año ${ }^{22,23}$. La mayor prevalencia se observa durante la edad pediátrica, no presenta diferencia de género ${ }^{1-8}$.

Existen diferentes teorías patogénicas ${ }^{1-7}$.

Teoría Genética, no la observamos, incluso dos pacientes fueron producto de embarazo gemelar, sus hermanos no presentaron la enfermedad $^{24}$.

Teoría Inmunológica, si bien no detectamos anticuerpos ni inmunocomplejos, todos los pacientes se beneficiaron con el uso de corticoides e inmunosupresores ${ }^{14-20}$.

Teoría Alérgica, la hipersensibilidad a la proteína de la leche de vaca y enfermedad celíaca, fueron descartadas en nuestros pacientes ${ }^{2-4}$.

Teoría Ambiental, la mitad de nuestros pacientes procedían de una misma zona rural con intensa actividad agrícola ${ }^{25,26}$, no encontramos diferencias significativas en relación a la evolución con el resto de los pacientes estudiados.

La HPI puede presentar la clásica tríada: hemoptisis, anemia e infiltrados pulmonares ${ }^{1-8}$, la que no siempre se encuentra completa. La hemoptisis puede ser masiva, produciendo, shock hipovolémico, anemia severa e insuficiencia respiratoria aguda, conduciendo eventualmente a un desenlace fatal, que en nuestra serie ocurrió en 2 casos. La hemoptisis no se presentó en 4 de nuestros pacientes. Sin embargo, el sangrado crónico persistió, manifestándose como una anemia de difícil manejo, incluso dos pacientes estuvieron en control en hematología presentando múltiples crisis de anemia (hemoglobina: $6 \mathrm{~g} / \mathrm{dL}$ ), llegando, en uno de ellos, hasta la postración, requiriendo múltiples transfusiones, en ambos se produjo retraso en el diagnóstico.

Durante la fase aguda los infiltrados pulmonares se presentan como imágenes algodonosas $\mathrm{y}$ vidrio esmerilado ${ }^{8-10}$, confundiéndose con neumonía y edema pulmonar agudo (Figuras 1A, 1B, $2 \mathrm{~A}, 2 \mathrm{~B}$ y $2 \mathrm{C}$ ), lo que también produjo latencia en el diagnóstico. Durante la fase crónica se observan infiltrados intersticiales reticulares y micro- 
Tabla 1. Datos demográficos, clínicos, evolución, radiológicos, función pulmonar y tratamiento

\begin{tabular}{|c|c|c|c|c|c|c|c|c|}
\hline & Sexo & $\begin{array}{l}\text { Edad } \\
\text { inicio } \\
\text { (años) }\end{array}$ & $\begin{array}{l}\text { Edad } \\
\text { actual } \\
\text { (años) }\end{array}$ & $\begin{array}{c}\text { Edad } \\
\text { mejoría } \\
\text { (años) }\end{array}$ & $\begin{array}{c}\text { TC } \\
\text { tórax } \\
\text { (patrón) }\end{array}$ & $\begin{array}{l}\text { Espirometría/ } \\
\text { pletismografía }\end{array}$ & $\begin{array}{c}\text { Tratamiento } \\
\text { inicial }\end{array}$ & $\begin{array}{l}\text { Tratamiento } \\
\text { mantención }\end{array}$ \\
\hline LT & M & 2 & 23 & 18 & 1 & $\begin{array}{c}\text { Obstructivo/ } \\
\text { atrapamiento aéreo }\end{array}$ & $\mathrm{HC} / \mathrm{MP}$ & $\mathrm{P}+\mathrm{A}$ \\
\hline $\mathrm{RM}$ & $\mathrm{F}$ & 13 & 25 & 15 & 0 & Normal/Normal & MP & $\mathrm{P}+\mathrm{A}$ \\
\hline $\mathrm{CR}$ & $\mathrm{F}$ & 12 & 17 & 13 & 0 & Normal/Normal & MP & $\mathrm{P}+\mathrm{A}$ \\
\hline FG & M & 2 & - & Fallecido & 2 & Restrictivo & MP & $\mathrm{P}+\mathrm{A}$ \\
\hline BG & $\mathrm{F}$ & 3 & 12 & Tratamiento & 1 & Normal/Normal & MP & $\mathrm{P}+\mathrm{A}$ \\
\hline $\mathrm{RP}$ & M & 15 & 17 & 16 & 3 & Normal/Normal & MP & $\mathrm{P}+\mathrm{A}$ \\
\hline $\mathrm{CB}$ & $\mathrm{F}$ & 13 & 31 & 20 & 0 & Normal & $\mathrm{P}$ & $\mathrm{P}+\mathrm{A}$ \\
\hline $\mathrm{PH}$ & $\mathrm{F}$ & 13 & 30 & 13 & 0 & Normal & $\mathrm{P}$ & $\mathrm{P}+\mathrm{A}$ \\
\hline PS & M & 2 & 24 & 18 & 2 & Restrictivo & $\mathrm{P}$ & $\mathrm{P}+\mathrm{A} / \mathrm{P}+\mathrm{H}$ \\
\hline $\mathrm{RJ}$ & M & 11 & 33 & 22 & 1 & Restrictivo & $\mathrm{P}$ & $\mathrm{P}+\mathrm{A}$ \\
\hline $\mathrm{CC}$ & $\mathrm{F}$ & 6 & - & Fallecida & 3 & - & $\mathrm{P}$ & $\mathrm{P}+\mathrm{A}$ \\
\hline FP & M & 8 & 11 & Tratamiento & 4 & Restrictivo & $\mathrm{P}$ & $\mathrm{P}+\mathrm{H} / \mathrm{P}+\mathrm{A} / \mathrm{P}+\mathrm{M}$ \\
\hline $\mathrm{NC}$ & M & 7 & 8 & Tratamiento & 0 & Normal & MP & $\mathrm{P}+\mathrm{A}$ \\
\hline
\end{tabular}

M: masculino; F: femenino; TC: Tomografía computada.

\section{Patrones TC de tórax}

0 : normal

1: infiltrado micronodular

2: infiltrado retículo/nodular

3: vidrio esmerilado

4: fibrosis

\section{Fármacos}

$\mathrm{HC}$ : hidrocortisona

MP: metilprednisolona

P: prednisona

A: azatioprina

$\mathrm{H}$ : hidoxicloroquina

M: micofenolato
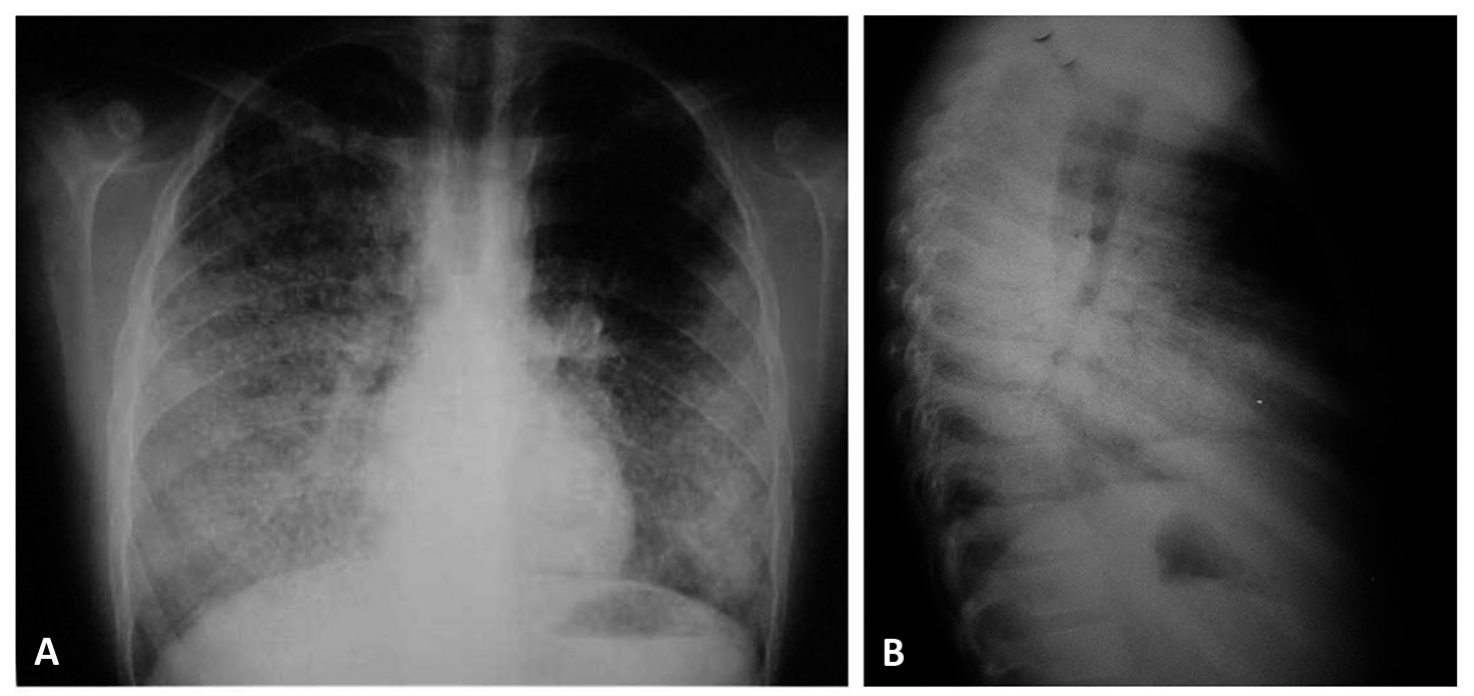

Figura 1A y 1B. Rx tórax (AP y lateral) (sangrado alveolar agudo sobre enfermedad pulmonar intersticial crónica): infiltrados algodonosos y micronodulares difusos bilaterales. Paciente RJ (niño de 15 años de edad). 


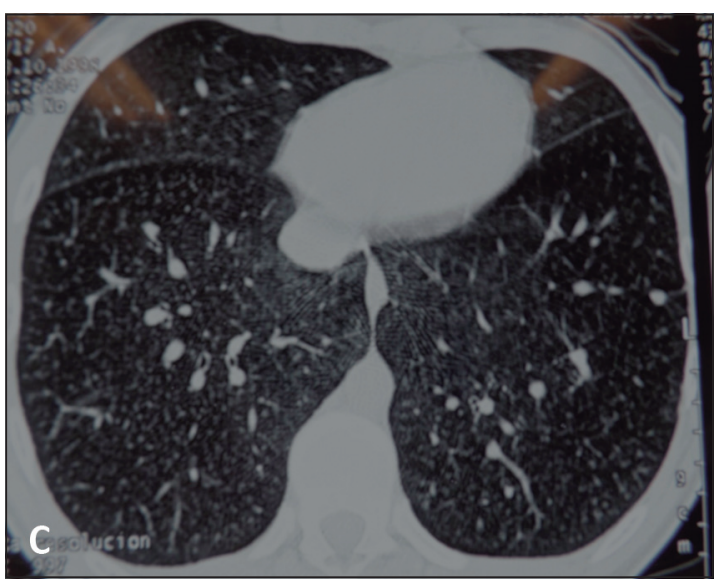

Figura 1C. TC tórax $\left(10^{\circ}\right.$ mes post sangrado alveolar): infiltrados intersticiales micronodulares difusos bilaterales. Paciente RJ (niño de 15 años de edad).

nodulares (Figuras 1A, 1B y 1C). Si los sangrados persisten pueden conducir a una enfermedad pulmonar intersticial y posteriormente a fibrosis pulmonar e insuficiencia respiratoria crónica, lo que ocurrió en uno de nuestros pacientes ${ }^{27}$.

El recuento de HSF obtenidos de preferencia en LBA, resulta fundamental para el diagnóstico; en un modelo murino los HSF aparecieron al $3^{\text {er }}$ día del sangrado, el mayor porcentaje se observó entre 6 y 10 días. En otro reporte, el hallazgo de $35 \%$ de HSF tuvo una sensibilidad de $100 \%$ y especificidad de $96 \%^{11,12}$. Todos los pacientes de este estudio presentaron HSF mayor de $30 \%$, obtenidos mediante LBA, cuando la muestra se consiguió a partir del cuarto día de sangrado. En dos pacientes cuyos LBA fueron realizados precozmente, los recuentos resultaron inicialmente negativos.

Se ha reportado capilaritis en la biopsia pulmonar en ausencia de vasculitis sistémica, esto nos hace replantear la indicación de biopsia ${ }^{4}$, a la que sólo recurrimos en un caso, la que fue diagnosticada como enfermedad pulmonar intersticial crónica.

El pronóstico es favorable si el diagnóstico y tratamiento se hace precozmente con corticoides más un inmunosupresor y corticoides inhala$\operatorname{dos}^{15-20}$, también lo es en niñas adolescentes, este último hecho ocurrió en 4 de ellas, las que evolucionaron con TC tórax y espirometrías normales. En cambio, los niños mostraron deterioro en la imagenología, incluso un paciente evolucionó a fibrosis y las espirometrías evidenciaron un patrón predominantemente restrictivo.

Tanto el tratamiento inicial con el uso de metilprednisolona en bolos diarios o prednisona ${ }^{28}$, como el de mantención con prednisona más un inmunosupresor fueron exitosos, elprimero controló la enfermedad en la fase aguda y el tratamiento de mantención disminuyó el número de reagudizaciones.

Este seguimiento confirma que la HPI es una enfermedad grave. Hubo recaídas serias, con uso de VMNI, VMI e incluso $\mathrm{VAFO}^{29} \mathrm{y}$ dos fallecimientos. Los dos niños fallecidos presentaron una reagudización intratable, debida en un caso a la suspensión del tratamiento y en el otro caso a una neumonía bacteriana grave intercurrente.

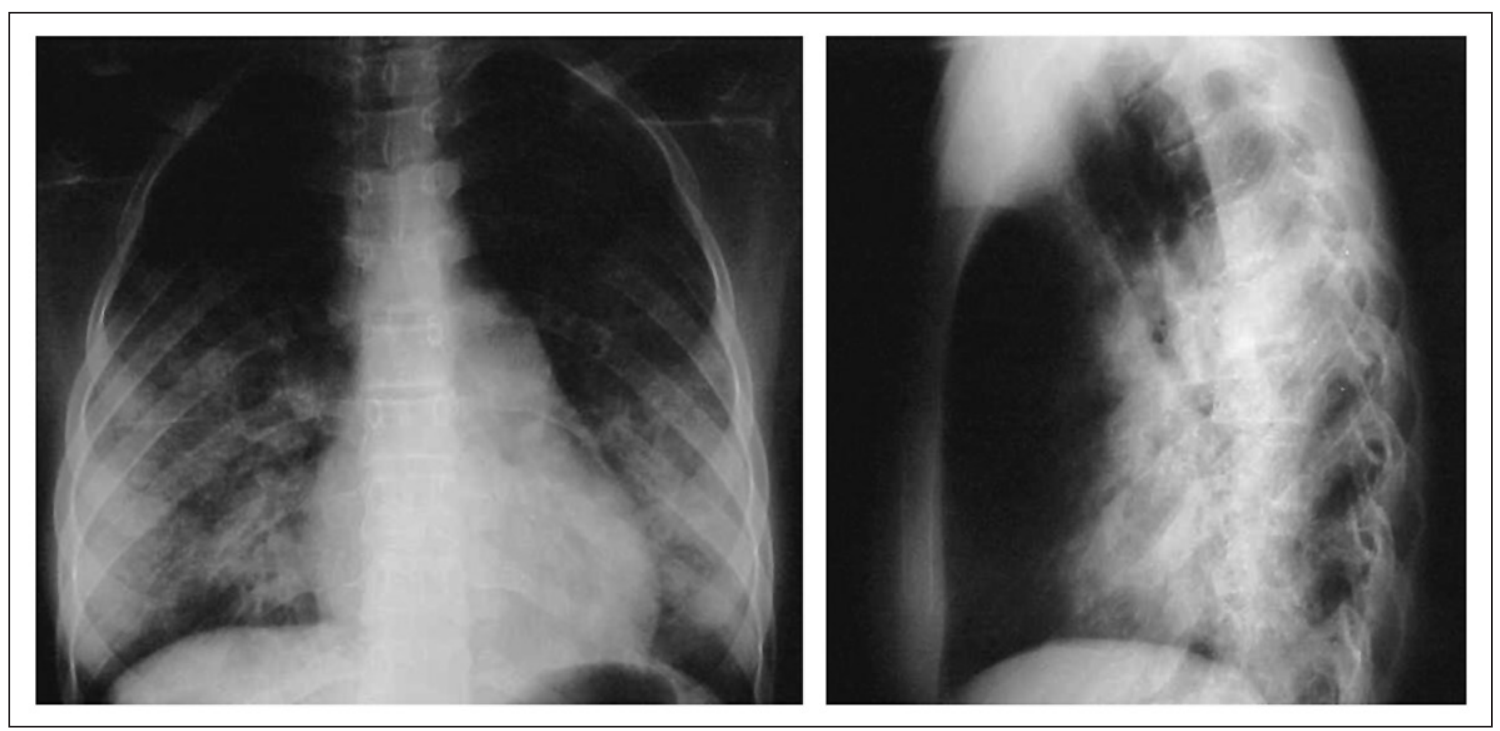

Figura 2A y 2B. Rx tórax (AP y lateral) (sangrado alveolar agudo): infiltrados algodonosos bibasales. Paciente CB (niña de 13 años de edad). 


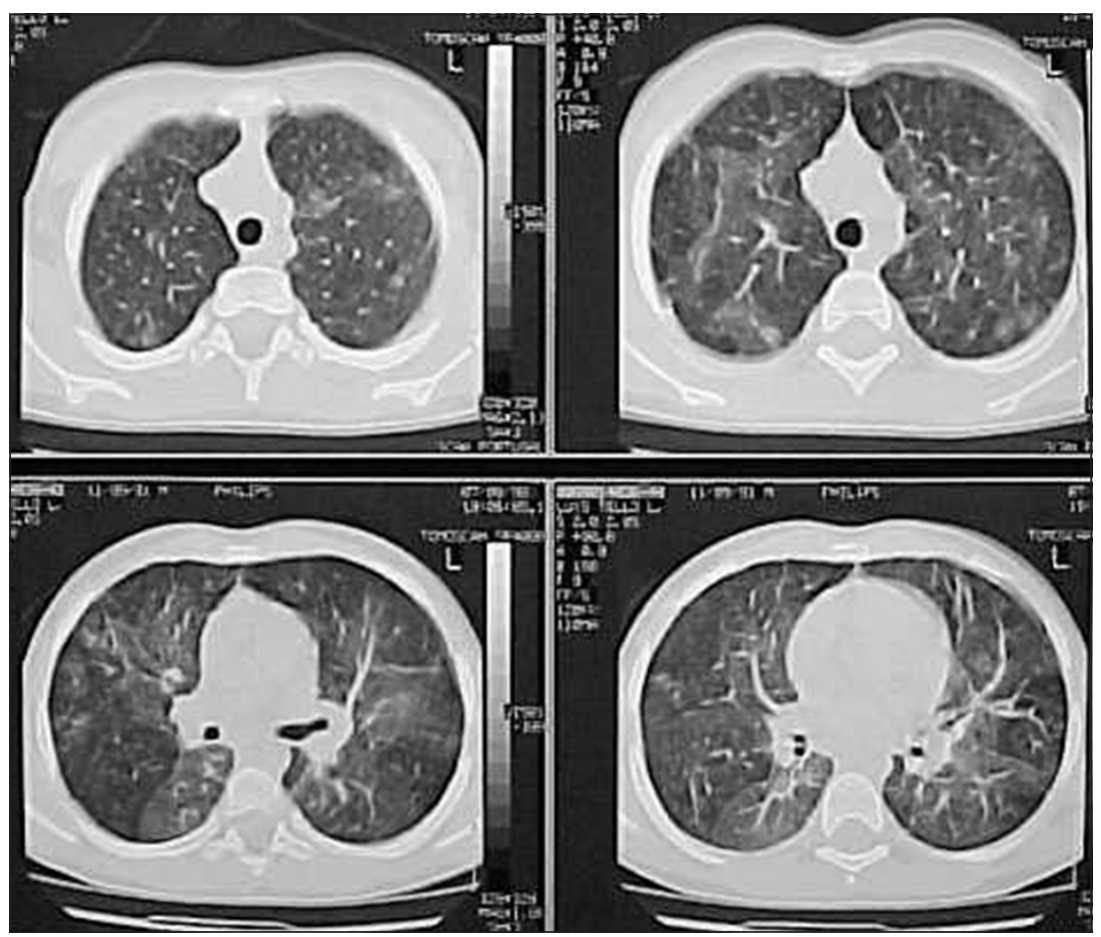

Figura 2C. TC tórax (sangrado alveolar agudo): infiltrados algodonosos y vidrio esmerilado bilateral. Paciente $\mathrm{CB}$ (niña de 13 años de edad).

Hubo remisiones de la enfermedad durante la adolescencia, hecho que no ha sido reportado.

La HPI en pediatría sería una enfermedad predominantemente transitoria, pero potencialmente fatal, lo que requiere que estos pacientes deban ser estrictamente controlados. Además, se debe estar atentos ante la posible aparición de alguna enfermedad autoinmune ${ }^{16}$.

Se reportaron 5 embarazos de curso fisiológico, los que presentaron escasos sangrados pulmonares durante el puerperio, hecho pocas veces descrito en la literatura ${ }^{30}$.

En el transcurso de este seguimiento se detectaron RAM en varios niños, la más frecuente fue Síndrome de Cushing asociado en dos niños a opacidades subcapsulares, y en un niño a diabetes por corticoides. Ninguna de estas RAM fue motivo de suspensión del tratamiento.

Finalmente, teniendo en cuenta la relación costo beneficio del tratamiento, las RAM observadas no resultaron ser severas.

\section{Bibliografía}

1.- IOACHIMESCU OC, SIEBER S, KOTCH A. Idiopathic pulmonary haemosiderosis revisited. Eur Respir J. 2004; 24: 162-70

2.- VECE T, MORALES DE GUZMÁN M, LANGS-
TON C, FAN LL. Diffuse Alveolar Hemorrhage in Children. En: Kendig E, Wilmott R, Li A, Deterning R, Ratjen F. Kendig's Disorders of the Respiratory Tract in Children. 9th Edition. Elsevier Inc. Philadelphia, Estados Unidos 2019; 61: 893-902e2.

3.- YAJUN ZHANG, FENGLAN LUO, NINI WANG, YUE SONG, YUHONG TAO. Clinical characteristics and prognosis of idiopathic pulmonary hemosiderosis in pediatric patients. Journal of International Medical Research 2018 47:1, 293-302.

4.- SUSARLA S, FAN LL. Diffuse alveolar hemorrhage syndromes in children. Current Opinion in Pediatrics 2007; 19: 314-20.

5.- YAO TC, HUNG IJ, WONG KS, HUANG JL, NIU CK. Idiopathic pulmonary hemosiderosis: An oriental experience. J Pediatr Child Health. 2003; 39: 27-30.

6.- KABRA SK, BHARGAVA S, LODHA R, SATYAVANI A, WALIA M. Idiopathic pulmonary hemosiderosis: Clinical Profile and Follow up of 26 children. Indian Pediatrics 2007; 44: 333-8.

7.- TAYTARD J, NATHAN N, DE BLIC J, FAYON M, EPAUD R. New insights pediatric idiophatic pulmonary hemosiderosis the French RespiRare ${ }^{\circledR}$ cohort. Orpahnet J Rare Dis. 2013; 8:161.

8.- MILMAN N, KING TE, HOLLINGSWORTH H. Idiopathic pulmonary hemosiderosis. UpToDate 2017; disponible en: https:/www.uptodate.com/contents/ idiopathic-pulmonary-hemosiderosis.

9.- ALBELDA SM, GEFTER WB, EPSTEIN DM, MI- 
LLER WT. Diffuse pulmonary hemorrhage: A review and classification. Radiology. 1985; 154: 289-97.

10.- AKYAR S, OZBEK SS. Computed tomography findings in idiopathic pulmonary hemosiderosis. Respiration 1993; 60: 63-4.

11.- SALIH Z, AKHTER A, AKTHTER J. Specificity and Sensitivity of Hemosiderin-Laden Macrophages in Routine Bronchoalveolar Lavage in Children. Arch Pathol Lab Med. 2006; 130; 1684-6.

12.- EPSTEIN C, ELIDEMIR O, COLASURDO G, FAN LL. Time Course of Hemosiderin Production by Alveolar Macrophages in a Murine Model. Chest 2001; 120 : 2013-20.

13.- SHERMAN JM, WINNIE G, TOMASEN MJ. Time course of hemosiderin production and clearance by human pulmonary macrophages. Chest. 1984; 86: 409.

14.- SAEED MM, WOO MS, MACLAUGHLIN EF. Prognosis in pediatric idiopathic pulmonary hemosiderosis. Chest 1999; 116: 721-5.

15.- BAGNATO L, GRILLI C, PORTIOLI P, BIELLA C, CARNELLI. Long-term evaluation of immunosuppressive therapy in childhood idiopathic pulmonary haemosiderosis. Pediatr Med Chir. 1986; 8: 671-4.

16.- Le Clainche L, Le Bourgeois M, Fauroux B. Long-term outcome of idiopathic pulmonary hemosiderosis in children. (Baltimore) Medicine 2000; 79: 318-26.

17.- ROSSI GA, BALZANO E, BATTISTINI E. Longterm prednisone and azathioprine treatment of a patient with idiopathic pulmonary hemosiderosis Pediat Pulmonol. 1992; 13: 176-80.

18.- KIPER N, GOCMEN A, OZCELIK U, DILBER E, ANADOL D. Long-term clinical course of patients with idiopathic pulmonary hemosiderosis (1979-1994): prolonged survival with low-dose corticosteroid therapy. Pediatr Pulmonol. 1999; 27: 180-4.

19.- ZAKI M, SALEH QA, MUTARI GA. Effectiveness of chloroquine therapy in idiopathic pulmonary hemosiderosis. Pediatr Pulmonol. 1995; 20: 125-6.

20.- GORAN E, ELINDER G. Budesonide inhalation to treat idiopathic pulmonary hemosiderosis. Lancet. 1985; 1: 981-2.

21.- CALABRESE F, GIACOMETTI C, REA F. Recurrence of idiopathic hemosiderosis in a young adult patient after bilateral single-lung transplantation. Transplantation 2002; 74: 1643-5.

22.- KJELLMAN B, ELINDER G, GARWICZ S, SVAN H. Idiopathic pulmonary haemosiderosis in Swedish children. Acta Paediatr Scand. 1984; 73: 584-8.

23.- OHGA S, TAKAHASHI N. Haemosiderosis in Japan: 39 possible cases from a survey questionnaire. Eur J Pediatr. 1995; 154: 994-5.

24.- BECKERMAN RC, TAUSSIG LM, PINNAS JL. Familial idiopathic pulmonary hemosiderosis. Am J Dis Child. 1979; 133: 609-11.

25.- CASSIMOS CD, CHRYSSANTHOPOULOS C, PANAGIOTIDOU C. Epidemiologic observations in idiopathic pulmonary haemosiderosis. J Pediatr. 1983; 102: 698-702.

26.- DEARBORN DG, SMITH PG, DAHMS B. Clinical profile of 30 infants with acute pulmonary hemorrhage in Cleveland. Pediatrics. 2002; 110: 627-37.

27.- BOCCON-GIBOD L, COUVREUR J. Results of lung biopsy in interstitial pneumopathies in children. A report on 100 cases. Ann Med Interne (Paris) 1979; 130: 501-6.

28.- OHGA S, NOMURA A, SUGA N, HIKINO S. Liposteroid against refractory pulmonary haemorrhage in idiopathic pulmonary haemosiderosis. Eur. J. Pediatr. 1994; 153: 687-90.

29.- SUN L, TSENG Y, HUANG S, HUANG P, KO W. Extracorporeal Membrane Oxygenation to rescue Profound Pulmonary Hemorrhage due to Idiopathic Pulmonary Hemosiderosis in a Child. Pediatr Pulmonol. 2006; 41: 900-3.

30.- MIRALlES F, TORRES J, ROBLES M. Pregnancy in patients with idiophatic pulmonary hemosiderosis. Presentation of a case. Arch Bronconeumol. 1998; 34 : 317-31.
Correspondencia a:

Dr. Julio Maggiolo M.

Hospital Exequiel González Cortés.

Unidad Broncopulmonar.

Gran Avenida 3300. San Miguel. Santiago, Chile.

Email: maggiolojulio@gmail.com 